\title{
LAYER UNDULATIONS IN SMECTIC A LIQUID CRYSTALS
}

\author{
CARLOS J. GARCíA-CERVERA* AND SOOKYUNG JOO ${ }^{\dagger}$
}

\begin{abstract}
We investigate instabilities of smectic A liquid crystals when a magnetic field is applied in the direction parallel to smectic layers. We use the Landau-de Gennes model of smectic A liquid crystals to characterize the critical magnetic field. When smectic A liquid crystals are confined between parallel plates, we derive analytic estimates for the magnetic field strength, at which the undeformed state loses its stability. We also present numerical simulations to confirm the Helfrich-Hurault effect due to the applied magnetic field.
\end{abstract}

1. Introduction. We consider smectic A liquid crystals confined in two flat plates and uniformly aligned in a way that the smectic layers are parallel to the bounding plates. If a magnetic field is applied in a direction parallel to the smectic layers, the instability occurs above the threshold magnetic field. When the magnetic field reaches the critical threshold, one can see periodic perturbation of the layers. This phenomenon is called the Helfrich-Hurault effect.(See [8] and [9].) We present the analytical estimate of the critical field and perform numerical simulations describing this effect by using the Landau-de Gennes model.

Liquid crystal phases form when a material has a degree of positional or orientational ordering yet stays in a liquid state. In the nematic state, molecules tend to align themselves along a preferred direction with no positional order of the centers of mass. The unit vector field $\mathbf{n}$, nematic director, represents the average direction of molecular alignment. Moreover, if the liquid crystal is chiral, $\mathbf{n}$ follows a helical pattern, with temperature-dependent pitch. Upon lowering the temperature, or increasing concentration, according to whether the liquid crystal is thermotropic or lyotropic, the nematic liquid crystal experiences a transition to the smectic A phase with molecules arranged along equally spaced layers. The molecules tend to align themselves along the direction perpendicular to the layers.

The Helfrich-Hurault effect in a lamellar system can be caused by magnetic/electric field or by mechanical tension [4]. In this paper, we study the magnetic field driven instabilities in smectic A liquid crystals. Helfrich and Hurault proposed the model that can explain the periodic perturbations in cholesteric liquid crystals under a magnetic field or an electric field applied parallel to the helical axis ([8], [9]). They assumed that the layers are fixed at the cell boundaries, i.e., the undulations vanish at the boundaries. Still with this assumption, Stewart extended the classic Helfrich-Hurault theory to three dimensional finite samples of smectic A liquid crystals in [13]. However, he made an ansatz to simplify the problem.

Experimental studies of undulations of two dimensional and three dimensional systems were performed in [10] and [12], respectively. They used cholesteric liquid crystals with a pitch $5 \mu m$ and $50-70 \mu m$ cell thickness for the optical study. Since the layer thickness of smectic A liquid crystals is in the nanometer range $(\mathrm{nm})$, it is too small to visualize the layer distortions. Their experiments show that there are layer undulations on the boundary of the sample. Motivated by the experimental result, Lavrentovich et al. proposed the model with weak anchoring condition so that the undulations are allowed to appear on the boundaries. By making an ansatz of

\footnotetext{
*Mathematics Department, University of California, Santa Barbara, CA 93106, (cgarcia@math.ucsb.edu).

${ }^{\dagger}$ Mathematics Department, University of California, Santa Barbara, CA 93106.(sjoo@math. ucsb.edu)
} 
periodic undulations, they show that their model explains the experiment better than the classic Helfrich-Hurault theory.

In section 2, we present the model and the geometry for our problem and state the existence result of minimizers of the free energy. The models used in previous works are based on an assumption that the director and the layer normal vector are identical, either in an infinite sample $([4],[8],[9])$, or in a finite sample [13]. We refine the theory to allow the director and the layer normal to differ by studying the Landau-de Gennes energy of smectic A liquid crystals. In [3], motivated from the analogy to the Ginzburg Landau model for superconductivy, de Gennes introduced the complex function to describe layer structures of smectic liquid crystals. This model was used in [1] to rigorously analyze the phase transition between the chiral nematic and smectic A liquid crystals. Recently, Lin and Pan used this model to show that critical magnetic field is achieved in an arbitrary domain [11]. However, they did not obtain the estimate of the critical magnetic field.

In section 3, we find the analytical estimate of the critical magnetic field for layer undulations. The theory of all previous works makes a choice of sinusoidal perturbation for the undulation in order to derive the critical magnetic field. Without this assumption, we derive the estimate of the threshold in terms of the cell thickness. More precisely, we prove that there exist universal constants $0<c_{1}<c_{2}$, such that, if $2 d$ is the cell thickness, then the critical field $H_{c}$ satisfies

$$
\left(\frac{c_{1} K}{\chi_{a} d \lambda}\right)^{\frac{1}{2}} \leq H_{c} \leq\left(\frac{c_{2} K}{\chi_{a} d \lambda}\right)^{\frac{1}{2}},
$$

where $K, \chi_{a}, \lambda$ are material constants, which will be discussed in section 2. This estimate is consistent with the result found in the classic Helfrich-Hurault theory (see p.363 of [4] and [9]). We should mention that the scaling of the critical field in this case is different from the threshold for Fredericks transition of nematic liquid crystals, where the critical field is proportional to $1 / d[16]$.

In section 4, We perform numerical simulations to the gradient flow equations. We find undulation instabilities above the critical magnetic field.

2. The model. The total free energy density of smectic A liquid crystals consists of the nematic $f_{n}$ and smectic $f_{s}$ part. The Oseen-Frank energy density for a nematic is given by

$$
\begin{aligned}
f_{n} & =K_{1}(\nabla \cdot \mathbf{n})^{2}+K_{2}(\mathbf{n} \cdot \nabla \times \mathbf{n})^{2}+K_{3}|\mathbf{n} \times(\nabla \times \mathbf{n})|^{2} \\
& +\left(K_{2}+K_{4}\right)\left(\operatorname{tr}(\nabla \mathbf{n})^{2}-(\nabla \cdot \mathbf{n})^{2}\right),
\end{aligned}
$$

where $K_{1}, K_{2}$ and $K_{3}$ are the splay, twist, and bend elastic constants, respectively. The last term in $f_{n}$ is a null-Lagrangian since its integral only depends on the boundary values of $\mathbf{n}$. We consider the energy with the one constant approximation case, $K_{1}=K_{2}=K_{3}=\frac{K}{2}>0$ and $K_{4}=0$. Then the nematic energy density becomes

$$
\frac{K}{2}|\nabla \mathbf{n}|^{2}
$$

In order to associate smectic and nematic structure with a state $(\mathbf{n}, \Psi)$ we write

$$
\Psi(\mathbf{x})=\rho(\mathbf{x}) e^{i \varphi(\mathbf{x})} .
$$

Then the molecular mass density is defined by

$$
\delta(\mathbf{x})=\rho_{0}(\mathbf{x})+\frac{1}{2}\left(\Psi(\mathbf{x})+\Psi^{*}(\mathbf{x})\right)=\rho_{0}(\mathbf{x})+\rho(\mathbf{x}) \cos \varphi(\mathbf{x}),
$$


where $\rho_{0}$ is a locally uniform mass density, $\rho(\mathbf{x})$ is the mass density of the smectic layers, and $\varphi$ parametrizes the layers, so that $\nabla \varphi$ is in the direction normal to the layer. Finally, $q$ is the wave number and $2 \pi / q$ is the layer thickness.

The Landau-de Gennes energy density for smectic A is given by

$$
f_{s}=\frac{C}{2}|\nabla \Psi-i q \mathbf{n} \Psi|^{2}+r|\Psi|^{2}+\frac{g}{2}|\Psi|^{4},
$$

where $C$ and $g$ are positive material constants, and $r$ may be positive or negative. In [1], the energy density $f_{n}+f_{s}$ was used to study the phase transition and stability of the equilibrium states. Since we investigate the smectic structure far from the nematic-smectic transition, we may assume that the magnitude of the smectic order parameter is constant, i.e., $\rho$ is constant. Then $f_{s}$ becomes

$$
f_{s}=\frac{C q^{2} \rho^{2}}{2}|\nabla \varphi-\mathbf{n}|^{2} .
$$

This energy density vanishes when $\nabla \varphi=\mathbf{n}$, which describes the configuration of smectic A liquid crystals.

The magnetic free energy density is given by $([4],[14])$

$$
f_{m}=-\frac{\chi_{a}}{2}(\mathbf{n} \cdot \mathbf{H})^{2}=-\frac{\chi_{a}}{2} \sigma^{2}(\mathbf{n} \cdot \mathbf{h})^{2},
$$

where $\chi_{a}$ is the magnetic anisotropy, $\mathbf{H}=\sigma \mathbf{h}$, and $\sigma=|\mathbf{H}|$. We assume that $\chi_{a}>0$. Therefore, the energy (2.1) favors molecular orientations where the director is parallel to the applied magnetic field.

Collecting all contributions to the free energy, the free energy density for the one-constant approximation model becomes

$$
f=\frac{K}{2}|\nabla \mathbf{n}|^{2}+\frac{B}{2}|\nabla \varphi-\mathbf{n}|^{2}-\frac{\chi_{a}}{2} \sigma^{2}(\mathbf{h} \cdot \mathbf{n})^{2}
$$

where $B=C q^{2} \rho^{2}$ is called the de Gennes compressibility constant.

In this paper, we consider a two dimensional domain

$$
\Omega=[-L, L] \times[-d, d] .
$$

We also assume that $\mathbf{h}=(1,0)$ so that the magnetic field tends to make the director orient along the $x$ direction. We impose the periodic boundary condition for $\phi$ and $\mathbf{n}$ in the $x$ direction so that we can minimize the unnecessary boundary effect, while we assume the strong anchoring condition for $\mathbf{n}$ on the boundary plates, i.e., simply

$$
\mathbf{n}(x, \pm d)=(0,1) \quad \text { for all } x \in[-L, L] .
$$

However, we do not impose any boundary conditions on $\phi$.

We make the problem dimensionless by introducing new variables

$$
\bar{x}=\frac{x}{\lambda}, \quad \bar{y}=\frac{y}{\lambda}, \quad \text { and } \quad \varphi=\lambda \phi,
$$

where $\lambda=\sqrt{\frac{K}{B}}$ is of the order of the smectic layer thickness. Then the free energy (2.2) becomes

$$
\frac{B}{2} \int_{\bar{\Omega}}\left(|\nabla \mathbf{n}|^{2}+(\nabla \phi-\mathbf{n})^{2}-\kappa(\mathbf{n} \cdot \mathbf{h})^{2}\right) d \overline{\mathbf{x}}
$$


where the dimensionless parameters are given by

$$
\kappa=\frac{\chi_{a} \sigma^{2}}{B}, \quad \bar{\Omega}=[-\bar{L}, \bar{L}] \times[-h, h], \quad h=\frac{d}{\lambda}, \quad \bar{L}=\frac{L}{\lambda} .
$$

Since $h$ is the ratio of the cell thickness to the layer thickness, we may assume that $h \gg 1$. In fact, the values $d=1 \mathrm{~mm}$ and $\lambda=20 \AA$ are employed in [4]. Then $h=5 \times 10^{5}$. In this paper, we will consider $h \geq 1$.

From $|\mathbf{n}|=1$, we can introduce the scalar variable $\theta$, with $0 \leq \theta<2 \pi$, such that

$$
\mathbf{n}=(\sin \theta, \cos \theta) .
$$

Then the free energy (2.4) becomes, dropping the bar notation,

$$
\left.\mathfrak{F}(\theta, \phi)=\int_{\Omega}\left(\phi_{x}-\sin \theta\right)^{2}+\left(\phi_{y}-\cos \theta\right)^{2}+|\nabla \theta|^{2}-\kappa \sin ^{2} \theta\right) d y d x
$$

and the corresponding boundary condition on $\theta$ is the homogeneous Dirichlet boundary condition on the top and the bottom of the plate. This energy (2.5) has a trivial critical point, $\theta=0, \phi=y$, which describes the undeformed state where the layers are parallel to the boundary plates and the directors are aligned in the $y$ direction. The second variation of the energy at the undeformed state, $\phi_{0}=y$, and $\theta_{0}=0$, gives

$$
\begin{aligned}
\frac{1}{2} D^{2} \mathfrak{F}\left(\theta_{0}+t \theta, \phi_{0}+t \phi\right) & :=\left.\frac{1}{2} \frac{d^{2}}{d t^{2}}\right|_{t=0} \mathfrak{F}\left(\theta_{0}+t \theta, \phi_{0}+t \phi\right) \\
& =\int_{\Omega}\left(\left(\phi_{x}-\theta\right)^{2}+\phi_{y}^{2}+|\nabla \theta|^{2}-\kappa|\theta|^{2}\right) d x d y
\end{aligned}
$$

The undeformed state, $\left(\theta_{0}, \phi_{0}\right)$, is stable if the second variation is nonnegative at $\left(\theta_{0}, \phi_{0}\right)$. Setting

$$
\mathcal{G}(\theta, \phi):=\int_{\Omega}\left(\left(\phi_{x}-\theta\right)^{2}+\phi_{y}^{2}+|\nabla \theta|^{2}\right) d x d y,
$$

the critical field $\kappa_{c}$ is defined by

$$
\kappa_{c}=\inf _{(\theta, \phi) \in \mathcal{A}} \mathcal{G}(\theta, \phi) .
$$

Here, the admissible set $\mathcal{A}$ is given by

$$
\begin{gathered}
\mathcal{A}=\left\{(\theta, \phi) \in W^{1,2}(\Omega) \times W^{1,2}(\Omega):\|\theta\|_{L^{2}(\Omega)}=1, \theta(x, \pm h)=0 \text { for all } x,\right. \\
\theta \text { and } \phi \text { periodic in the } x \text { direction }\}
\end{gathered}
$$

Thus, the undeformed state, $\left(\theta_{0}, \phi_{0}\right)$, is stable if $\kappa \leq \kappa_{c}$ and unstable if $\kappa>\kappa_{c}$. In section 3 of [11], they proved that the critical field $\kappa_{c}$ is achieved when Dirichlet boundary condition is imposed on $\partial \Omega$. Following their work, we consider $\phi$ as a function of $\theta$ and apply the standard calculus of variations to prove the existence of a minimizer of $\mathcal{G}$. For the reader's convenience, we present a short proof here.

Proposition 2.1. There exists a minimizer for $\mathcal{G}$ in $\mathcal{A}$.

Proof. If $(\theta, \phi)$ is a minimizer, then $\phi$ can be expressed in terms of $\theta$. That is, we write

$$
\begin{gathered}
\kappa_{c}=\inf \left\{\mathcal{G}\left(\theta, \phi_{\theta}\right): \theta \in W^{1,2}(\Omega),\|\theta\|_{L^{2}(\Omega)}=1, \theta(x, \pm h)=0 \text { for all } x\right. \\
\theta(-L, y)=\theta(L, y) \text { for all } y\}
\end{gathered}
$$


where $\phi_{\theta}$ is the solution of

$$
\begin{aligned}
& \Delta \phi_{\theta}=\frac{\partial \theta}{\partial x}, \quad \int_{\Omega} \phi_{\theta}=0 \\
& \frac{\partial \phi_{\theta}}{\partial y}(x, \pm h)=0 \text { for all } x, \quad \phi(-L, y)=\phi(L, y) \text { for all } y
\end{aligned}
$$

Now we take a minimizing sequence $\left\{\theta_{j}\right\}$ for $\mathcal{G}$ and write $\phi_{\theta_{j}}=\phi_{j}$. Since $\theta_{j}$ is bounded in $W^{1,2}(\Omega)$, it follows, for a subsequence, still labeled $\theta_{j}$ that

$$
\theta_{j} \rightarrow \theta_{\infty} \text { in } W^{1,2}(\Omega), \quad \theta_{j} \rightarrow \theta_{\infty} \text { almost everywhere in } \Omega .
$$

For the $L^{2}$ convergence on the boundary, we used the estimate, from the proof of Theorem 1.5.1.10 in [7],

$$
\int_{\partial \Omega}|u|^{2} d \sigma \leq C(\Omega)\left\{\|u\|_{L^{2}(\Omega)}\|\nabla u\|_{L^{2}(\Omega)}+\|u\|_{L^{2}(\Omega)}^{2}\right\},
$$

valid for all $u \in W^{1,2}(\Omega)$. We also have

$$
\int_{\Omega}\left(\frac{\partial \phi_{j}}{\partial x}-\theta_{j}\right)^{2}+\int_{\Omega}\left(\frac{\partial \phi_{j}}{\partial y}\right)^{2} \leq C
$$

for some constant $C$. Then together with $\int_{\Omega} \phi_{j}=0$, we have $\left\|\phi_{j}\right\|_{W^{1,2}(\Omega)} \leq C$. The elliptic estimates on (2.9) with $\theta=\theta_{j}$ gives $\left\|\phi_{j}\right\|_{W^{2,2}(\Omega)} \leq C$ and thus it follows, for a subsequence, still labeled $\phi_{j}$ that

$$
\phi_{j} \rightarrow \phi_{\infty} \text { in } W^{2,2}(\Omega), \quad \phi_{j} \rightarrow \phi_{\infty} \text { in } W^{1,2}(\Omega),
$$

and $\phi_{\infty}$ satisfies $(2.9)$ with $\theta=\theta_{\infty}$, i.e., $\phi_{\infty}=\phi_{\theta_{\infty}}$. Thus $\left(\theta_{\infty}, \phi_{\infty}\right) \in \mathcal{A}$ and

$$
\mathcal{G}\left(\theta_{\infty}, \phi_{\theta_{\infty}}\right) \leq \liminf _{j \rightarrow \infty} \mathcal{G}\left(\theta_{j}, \phi_{\theta_{j}}\right)=\inf _{\theta \in \mathcal{A}} \mathcal{G}\left(\theta, \phi_{\theta}\right)
$$

3. Characterization of the critical field. In this section, we prove that the critical field in (2.8) satisfies $\kappa_{c} \sim 1 / h$. In other words, we prove

Theorem 3.1. Assume that $L \geq h \geq 1$. Then, there exist universal constants $c_{1}$ and $c_{2}$ such that

$$
\frac{c_{1}}{h} \leq \kappa_{c} \leq \frac{c_{2}}{h}
$$

Note that this statement is equivalent to (1.1), where the estimate is expressed in terms of the real parameters.

We prove this theorem in two steps: In the first step, we prove a lower bound for the energy with the above-mentioned scaling. In the second step, we prove a matching upper bound.

The periodic boundary conditions allow us to use the Fourier series representation.

$$
\theta(x, y)=\sum_{n=-\infty}^{\infty} \theta_{n}(y) e^{i \mu_{n} x} \quad \text { and } \quad \phi(x, y)=\sum_{n=-\infty}^{\infty} \phi_{n}(y) e^{i \mu_{n} x}
$$


where $\mu_{n}=2 \pi n / L$. Then $(2.7)$ becomes

$$
\mathcal{G}(\theta, \phi)=2 L \int_{-h}^{h}\left(\sum_{n=-\infty}^{\infty}\left(\left|\theta_{n}^{\prime}\right|^{2}+\mu_{n}^{2}\left|\theta_{n}\right|^{2}+\left|\theta_{n}-i \mu_{n} \phi_{n}\right|^{2}+\left|\phi_{n}^{\prime}\right|^{2}\right)\right) d y .
$$

We define

$$
\begin{aligned}
\kappa(\mu) & =\inf _{(\theta, \phi) \in \mathcal{B}} \int_{-h}^{h}\left(\left|\theta^{\prime}\right|^{2}+\mu^{2}|\theta|^{2}+|\theta-i \mu \phi|^{2}+\left|\phi^{\prime}\right|^{2}\right) d y \\
& :=\inf _{(\theta, \phi) \in \mathcal{B}} \mathcal{F}(\theta, \phi, \mu),
\end{aligned}
$$

where

$$
\mathcal{B}=\left\{(\theta, \phi) \in W_{0}^{1,2}(-h, h) \times W^{1,2}(-h, h): \int_{-h}^{h}|\theta(y)|^{2} d y=1\right\} .
$$

As in [2], it follows that

$$
\begin{aligned}
\mathcal{G}(\theta, \phi) & \geq 2 L \sum_{n=-\infty}^{\infty} \kappa\left(\mu_{n}\right) \int_{-h}^{h}\left|\theta_{n}(y)\right|^{2} d y \\
& \geq 2 L \sum_{n=-\infty}^{\infty} \kappa_{c} \int_{-h}^{h}\left|\theta_{n}(y)\right|^{2} d y \\
& =\kappa_{c} \int_{\Omega}|\theta|^{2} d x d y
\end{aligned}
$$

for all $\theta \in W^{1,2}(\Omega)$. Immediately we see that

$$
\kappa_{c}=\inf _{\mu} \kappa(\mu)
$$

3.1. Lower bound. In following lemma we prove that there exists a constant $c_{1}$ such that $\kappa(\mu) \geq c_{1} / h$ for any $\mu$. This implies $\kappa_{c} \geq c_{1} / h$ due to (3.3). As a result, the undeformed state is stable if $\kappa \leq c_{1} / h$.

Lemma 3.2. Let $h>1$ and $\mu$ be given positive constants. Then there exists a universal positive constant $c_{1}$ such that

$$
\int_{-h}^{h}\left(|\theta-i \mu \phi|^{2}+\mu^{2}|\theta|^{2}+\left|\theta^{\prime}\right|^{2}+\left|\phi^{\prime}\right|^{2}\right) d y \geq \frac{c_{1}}{h} \int_{-h}^{h}|\theta|^{2} d y
$$

for all $(\theta, \phi) \in \mathcal{B}$.

Proof. We may assume that $\mu \neq 0$, otherwise the inequality (3.4) is trivial with $c_{1}=1$. By using

$$
\tilde{y}=\frac{y}{h}, \quad \text { and } \quad \varphi=i \mu \phi,
$$

the claim (3.4) is equivalent to

$$
\int_{-1}^{1}\left(\frac{1}{h}\left|\theta^{\prime}\right|^{2}+\frac{1}{h \mu^{2}}\left|\varphi^{\prime}\right|^{2}+h \mu^{2}|\theta|^{2}+h|\theta-\varphi|^{2}\right) d y \geq c_{1} \int_{-1}^{1}|\theta|^{2} d y .
$$


The Euler-Lagrange equation for $\varphi$ is

$$
\begin{aligned}
-\frac{1}{h^{2} \mu^{2}} \varphi^{\prime \prime}+\varphi & =\theta, \\
\varphi^{\prime}( \pm 1) & =0 .
\end{aligned}
$$

We will prove the inequality (3.5) for all $\theta$ and $\varphi$ satisfying (3.6). We suppose that this inequality is false, i.e., there exists $\left\{C_{n}\right\}$ with $\lim _{n \rightarrow \infty} C_{n}=0$, for which we can find $h_{n}, \mu_{n}, \varphi_{n} \in \mathcal{H}^{1}(-1,1)$ satisfying (3.6) and $\theta_{n} \in \mathcal{H}_{0}^{1}(-1,1)$ with $\left\|\theta_{n}\right\|_{2}=1$ for each $n=1,2, \ldots$ such that

$$
\int_{-1}^{1}\left(\frac{1}{h_{n}}\left|\theta_{n}^{\prime}\right|^{2}+\frac{1}{h_{n} \mu_{n}^{2}}\left|\varphi_{n}^{\prime}\right|^{2}+h_{n} \mu_{n}^{2}\left|\theta_{n}\right|^{2}+h_{n}\left|\theta_{n}-\varphi_{n}\right|^{2}\right) d y \leq C_{n}
$$

Then we have two small parameters,

$$
h_{n} \mu_{n}^{2} \leq C_{n} \quad \text { and } \quad 1 / h_{n} \leq C_{n}
$$

where the second inequality follows from the Poincaré inequality. We integrate the equation (3.6) to obtain

$$
\overline{\varphi_{n}}=\overline{\theta_{n}}
$$

where $\overline{\varphi_{n}}$ and $\overline{\theta_{n}}$ are averages of $\varphi_{n}$ and $\theta_{n}$, respectively. In the following proof, we denote by $C$ a universal positive constant which may differ from line to line. Also, by $C_{n}$ we denote $C \cdot C_{n}$. From the second and the last terms in (3.7) and the Poincaré inequality, we have

$$
\begin{aligned}
\frac{C_{n}}{h_{n}} & \geq \int_{-1}^{1}\left(\theta_{n}-\varphi_{n}\right)^{2}=\int_{-1}^{1}\left(\theta_{n}-\overline{\theta_{n}}-\left(\overline{\varphi_{n}}-\overline{\varphi_{n}}\right)\right)^{2} d y \\
& \geq \frac{1}{2} \int_{-1}^{1}\left(\theta_{n}-\overline{\theta_{n}}\right)^{2} d y-\int_{-1}^{1}\left(\varphi_{n}-\overline{\varphi_{n}}\right)^{2} d y \\
& \geq \frac{1}{2} \int_{-1}^{1}\left(\theta_{n}-\overline{\theta_{n}}\right)^{2} d y-C \int_{-1}^{1}\left(\varphi_{n}^{\prime}\right)^{2} d y \\
& \geq \frac{1}{2} \int_{-1}^{1}\left(\theta_{n}-\overline{\theta_{n}}\right)^{2} d y-h_{n} \mu_{n}^{2} C_{n} .
\end{aligned}
$$

Thus, we have

$$
\int_{-1}^{1}\left(\theta_{n}-\overline{\theta_{n}}\right)^{2} d y \leq C_{n}\left(\frac{1}{h_{n}}+h_{n} \mu_{n}^{2}\right)
$$

Using (3.11), together with (3.10), we obtain

$$
\int_{-1}^{1}\left(\varphi_{n}-\overline{\varphi_{n}}\right)^{2} d y \leq C_{n}\left(\frac{1}{h_{n}}+h_{n} \mu_{n}^{2}\right) .
$$

Since $\left\|\theta_{n}\right\|_{L^{2}}=1$, we have

$$
\int_{-1}^{1}\left(\theta_{n}-\overline{\theta_{n}}\right)^{2} d y=1-2{\overline{\theta_{n}}}^{2} .
$$


Together with (3.11), we see that ${\overline{\theta_{n}}}^{2} \rightarrow 1 / 2$ as $n \rightarrow \infty$. By taking a subsequence, still labeled with $n$, we may assume $\overline{\theta_{n}} \rightarrow 1 / \sqrt{2}$ as $n \rightarrow \infty$. Therefore, from (3.8), (3.9), and (3.12), we have

$$
\left\|\eta_{n}\right\|_{W^{1,2}}^{2} \leq C_{n}\left(\frac{1}{h_{n}}+h_{n} \mu_{n}^{2}\right) \leq C_{n}^{2}
$$

where $\eta_{n}=\varphi_{n}-1 / \sqrt{2}$. This implies

$$
\sup _{[-1,1]}\left|\eta_{n}\right| \leq C_{n}
$$

Setting $f_{n}=\theta_{n}-\eta_{n}$ and $\varepsilon_{n}=1 / h_{n} \leq C_{n}$, we rewrite the first and the last terms of (3.7) as

$$
\frac{1}{\varepsilon_{n}} \int_{-1}^{1}\left(f_{n}-\frac{1}{\sqrt{2}}\right)^{2} d y+\varepsilon_{n} \int_{-1}^{1}\left(f_{n}^{\prime}+\eta_{n}^{\prime}\right)^{2} d y \leq C_{n}
$$

where $\eta_{n}$ satisfies the estimate (3.13). The part on the left hand side is an Allen-Cahn functional with a single well potential. From the first integral in (3.15), one can see that for each $n$, there exists $a_{n} \in(-1,1)$ such that $1 / 2<\left|f_{n}\left(a_{n}\right)\right|<1$. Finally we get, from (3.15),

$$
\begin{aligned}
C_{n} & \geq \int_{-1}^{1}\left|f_{n}-\frac{1}{\sqrt{2}}\right|\left|f_{n}^{\prime}+\eta_{n}^{\prime}\right| d y \\
& \geq \int_{-1}^{1}\left|f_{n}-\frac{1}{\sqrt{2}}\right|\left|f_{n}^{\prime}\right| d y-\int_{-1}^{1}\left|f_{n}-\frac{1}{\sqrt{2}}\right|\left|\eta_{n}^{\prime}\right| d y \\
& \geq\left|\int_{-1}^{a_{n}}\left(f_{n}-\frac{1}{\sqrt{2}}\right) f_{n}^{\prime} d y\right|-\left\|f_{n}-\frac{1}{\sqrt{2}}\right\|_{L^{2}}\left\|\eta_{n}^{\prime}\right\|_{L^{2}},
\end{aligned}
$$

where we used the triangle inequality and Hölder's inequality. Doing the change of variables $r=f_{n}(y)$, it follows from (3.13) and (3.15) that

$$
\left|\int_{-\eta_{n}(-1)}^{f_{n}\left(a_{n}\right)}\left(r-\frac{1}{\sqrt{2}}\right) d r\right|-C_{n} \sqrt{C_{n} \varepsilon_{n}} \leq C_{n}
$$

This is a contradiction, since $1 / 2<\left|f_{n}\left(a_{n}\right)\right|<1$ and $\left|\eta_{n}(-1)\right| \leq C_{n}$, which follows from (3.14). In fact, computing the integral in (3.16) we obtain

$$
\begin{aligned}
C_{n}\left(1+\sqrt{C_{n} \varepsilon_{n}}\right) & \geq\left|\left(f_{n}\left(a_{n}\right)+\eta_{n}(-1)\right)\left(f_{n}\left(a_{n}\right)+\eta_{n}(-1)-\sqrt{2}\right)\right| \\
& \geq\left(\frac{1}{2}-C_{n}\right)\left(\sqrt{2}-1-C_{n}\right),
\end{aligned}
$$

which is impossible since $C_{n} \rightarrow 0$.

3.2. Upper bound. We find an upper bound for the critical field by evaluating (2.2) for an appropriate test function. Specifically, we prove that $\kappa \leq c_{2} / h$ for some $c_{2}>0$. This implies that if $\kappa>c_{2} / h$, the undeformed state, $\left(\theta_{0} \equiv 0, \phi_{0}=y\right)$, is no longer stable. It follows from (3.3) that it suffices to find an upper bound for the one-dimensional problem. That is the content of the following lemma. 
Lemma 3.3. Let $L \geq h \geq 1$. There exists a constant $c_{2}$ such that

$$
\mathcal{F}(\tilde{\theta}, \tilde{\phi}, \tilde{\mu}) \leq \frac{c_{2}}{h} \int_{-h}^{h}|\tilde{\theta}|^{2} d y
$$

for some $(\tilde{\theta}, \tilde{\phi}) \in \mathcal{B}$ and for some constant $\tilde{\mu}$.

Proof. Define $\tilde{\theta}, \tilde{\phi}$ and $\tilde{\mu}$ by

$$
\begin{aligned}
\tilde{\mu} & =\frac{2 \pi}{L} n_{0} \equiv \frac{2 \pi}{L}\left[\frac{L}{\sqrt{h}}\right], \\
\tilde{\theta} & =\cos \frac{\pi}{2 h} y, \\
\tilde{\phi} & =-\frac{i}{\mu} \cos \frac{\pi}{2 h} y,
\end{aligned}
$$

where [.] denotes the greatest integer function. Since $L \geq h \geq 1$, we may see that $n_{0} \geq 1$ and then

$$
n_{0}^{2} \leq \frac{L^{2}}{h} \leq\left(n_{0}+1\right)^{2} \leq 4 n_{0}^{2} .
$$

A simple computation shows that

$$
\mathcal{F}(\tilde{\theta}, \tilde{\phi}, \tilde{\mu}) \leq \frac{1}{h}\left(4 \pi^{2}+\frac{1}{4}+\frac{\pi^{2}}{4 h}\right) \int_{-h}^{h}|\tilde{\theta}(y)|^{2} d y .
$$

The lemma is proved with $c_{2}=4 \pi^{2}+1 / 4+\pi^{2} / 4$.

Theorem 3.1 is a consequence of the two lemmas 3.2 and 3.3 .

4. Numerical simulations. We have carried out two-dimensional simulations with model (2.2) to study the Helfrich-Hurault effect. The energy is minimized by solving the gradient flow:

$$
\begin{aligned}
& \frac{\partial \phi}{\partial t}=\Delta \phi-\nabla \cdot \mathbf{n} \\
& \frac{\partial \mathbf{n}}{\partial t}=\Pi_{\mathbf{n}}(\Delta \mathbf{n}+\nabla \phi-\mathbf{n}+\kappa(\mathbf{n} \cdot \mathbf{h}) \mathbf{h})
\end{aligned}
$$

where $\Pi_{n}(f)=f-(\mathbf{n}, f) \mathbf{n}$ is the projection onto the plane orthogonal to $\mathbf{n}$, and $(\mathbf{n}, f)$ denotes the usual $L_{2}$ inner product. This projection appears as a result of the constraint $|\mathbf{n}|=1$. For numerical purposes, it is more convenient to write this term as

$$
\frac{\partial \mathbf{n}}{\partial t}=-\mathbf{n} \times(\mathbf{n} \times(\Delta \mathbf{n}+\nabla \phi-\mathbf{n}+\kappa(\mathbf{n} \cdot \mathbf{h}) \mathbf{h})) .
$$

Written in this way, the equation resembles the Landau-Lifshitz equation of micromagnetics in the high damping limit [5], and the heat-flow of harmonic maps [15].

For the initial condition, we take a small perturbation from the undeformed state. More precisely, for all $(x, y) \in \Omega$,

$$
\begin{aligned}
& \mathbf{n}(x, y, 0)=\frac{\left(\epsilon u_{1}, 1+\epsilon u_{2}\right)}{\left|\left(\epsilon u_{1}, 1+\epsilon u_{2}\right)\right|}, \\
& \phi(x, y, 0)=y+\epsilon \phi_{0},
\end{aligned}
$$



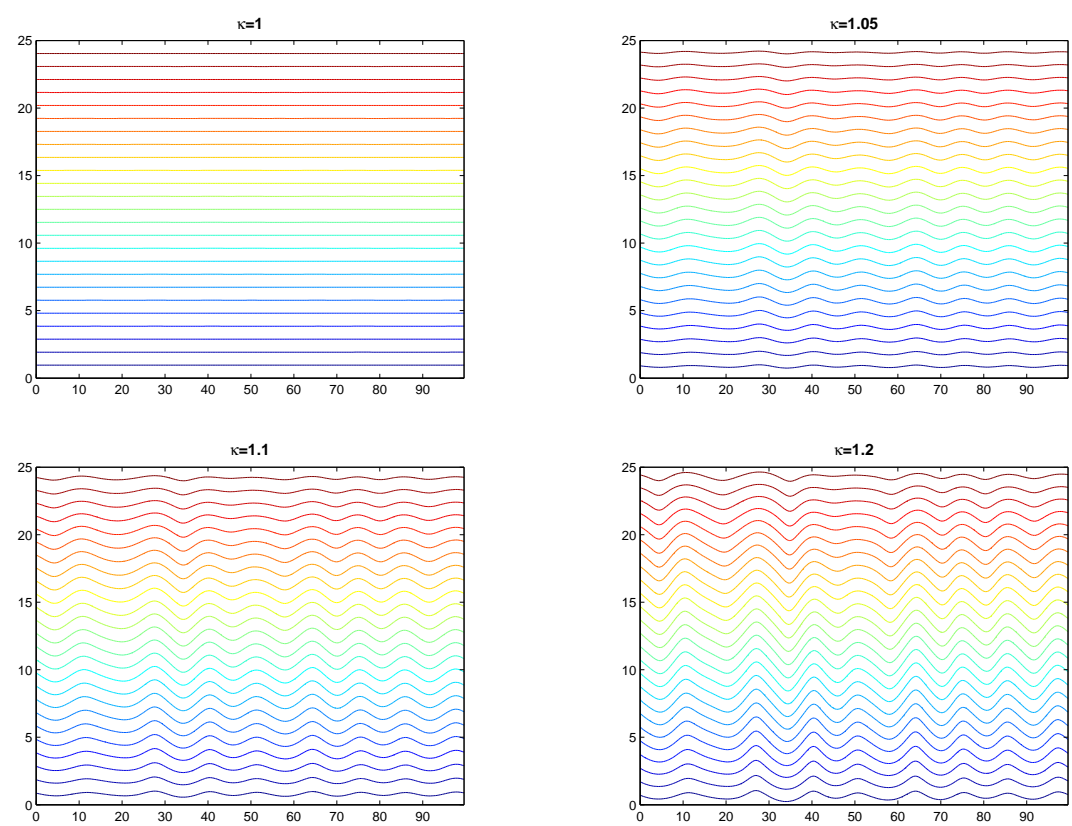

FIG. 4.1. Contour plots of $\phi$, the solution of the system (4.1).

where a small number $\epsilon=0.001$ and $u_{1}, u_{2}$, and $\phi_{0}$ are arbitrarily chosen. As described in section 2, we impose strong anchoring condition for the director field, (2.3), and natural boundary condition on $\phi$ at the top and the bottom plates,

$$
\left.\frac{\partial \phi}{\partial \nu}\right|_{y= \pm h}=\left.\mathbf{n} \cdot \nu\right|_{y= \pm h}
$$

Periodic boundary conditions are imposed for both $\mathbf{n}$ and $\phi$ on each side of the domain.

We use a Fourier spectral discretization in the $x$ direction, and second order finite differences in the $y$ direction. The fast Fourier transform is computed using the FFTW libraries [6]. We use the fourth order Runge-Kutta method to solve the corresponding initial value problem.

We take the domain size $L=100$ and $h=25$. A more physically relevant value for $h$ in smectic A liquid crystals is $5 \times 10^{5}$. However, the layer undulations can be observed if $h \gg 1$. In fact, the undulations in cholesteric liquid crystals occur with $h \approx 10([12])$. The numbers of grid points in the $x$ and $y$ directions are 256 and 128, respectively.

In Fig. 4.1 we show the layer structures in response to the various magnetic field strengths $\kappa$. The pictures are contour maps of $\phi$ since the level sets of $\phi$ represent the layer. One can see that the undeformed state is stable before the magnetic field $\kappa$ reaches the threshold $\kappa_{c}$. If $\kappa$ increases and reaches $\kappa_{c}$, the layer undulations occur. As $\kappa$ increases beyond $\kappa_{c}$, the displacement amplitude increases as in the Fig. 4.1. The maximum undulation occurs in the middle of the cell $(y=0)$ and the displacement amplitude decreases as approaching the boundary $(y= \pm h)$. In the classic HelfrichHurault theory, the layers are fixed at the boundary, i.e., no undulations at $y= \pm h$. However, Fig. 4.1 indicates that the undulations do not vanish at the boundary even though we impose the strong anchoring condition. 
5. Acknowledgements. The work of Carlos J. García-Cervera is supported by NSF grant DMS-0505738.

\section{REFERENCES}

[1] P. Bauman, M. Carme Calderer, C. Liu and D. Phillips The Phase Transition between Chiral Nematic and Smectic A* Liquid Crystals, Arch. Rational mech. Anal. 165 (2002), 161-186.

[2] P. Bauman and D. Phillips and Q. Tang Stable nucleation for the Ginzburg-Landau system with an applied magnetic field, Arch. Rational mech. Anal. 142 (1998), 1-43.

[3] P.G. De Gennes An analogy between superconductivity and smectics A, Solid State Commum. 10, (1972), 753-756 .

[4] P.G. De Gennes and J. Prost The Physics of Liquid Crystals, 2nd Edition. Clarendon Press, Oxford, 1993.

[5] W. E and X. Wang Numerical methods for the Landau-Lifshitz equation, SIAM J. Numer. Anal. 38, (2000), no. 5, 1647-1665.

[6] M. Frigo and S. G Johnson The design and implementation of FFTW3, Proc. of the IEEE 93, (2005), no. 2, 216-231, special issue on "Program Generation, Optimization, and Platform Adaptation"

[7] P. Grisvard Elliptic Problems in Nonsmooth Domains, Pitman Advanced Publishing Program, 1985.

[8] W. Helfrich Electrohydrodynamic and dielectric instabilities of cholesteric liquid cyrstals, J. Chem. Phys. 55, (1971), 839-842.

[9] J.P. Hurault Static distortions of a cholesteric planar structure induced by magnetic or ac electric-fields, J. Chem. Phys. 59, (1973), 2068-2075.

[10] T. Ishikawa and O.D. Lavrentovich Undulations in a confined lamellar system with surface anchoring, Phys. Rev. E 63 (2001), 030501(R).

[11] F.H. Lin and X.B. Pan Magnetic field-induced instabilities in liquid crystals, Siam J. Math. Anal. 38 (2007), no. 5, 1588-1612.

[12] B.I. Senyuk, I.I. Smalyukh and O.D. Lavrentovich Undulations of lamellar liquid crystals in cells with finite surface near and well above the threshold, Phys. Rev. E. 74 (2006), 011712.

[13] I.W. Stewart Layer undulations in finite samples of smectic A liquid crystals subjected to uniform pressure and magnetic fields, Phys. Rev. E. 58, (1998), no. 5, 5926-5933.

[14] I.W. Stewart The static and dynamic continuum theory of liquid crystals, Taylor \& Francis, 2004.

[15] M. Struwe Heat-flow methods for harmonic maps of surfaces and applications to free boundary problems, Springer, Lect. Notes Math. Berlin, 1324, (1988), 293-319.

[16] E.G. Virga Variational theories for liquid crystals, Chapman \& Hall, 1994. 